\title{
Suppression of esophageal squamous cell carcinoma development by mechanosensitive protein Piezo1 downregulation
}

Lu Gao ${ }^{1}$, Yun Ji ${ }^{1}$, Lulu Wang ${ }^{1}$, Meixia He${ }^{1}$, Xiaojing Yang ${ }^{1}$, Yibing Qiu ${ }^{1}$, Xu Sun ${ }^{2}$, Zhenyu Ji ${ }^{1}$, Guanrui Yang ${ }^{1}$, Jianying Zhang ${ }^{1}$, Shanshan Li $^{3}$, Liping Dai ${ }^{1}$, Liguo Zhang ${ }^{1}$ *

${ }^{1}$ BGI College \& Henan Institute of Medical and Pharmaceutical Sciences, Zhengzhou University, Zhengzhou 450052, China

2 Integrated TCM and Western Medicine Department, Affiliated Cancer Hospital of Zhengzhou University, Zhengzhou 450008, China

${ }^{3}$ Pathology Department, First Affiliated Hospital of Zhengzhou University, Zhengzhou University, Zhengzhou 450052, China

* Corresponding author: Liguo Zhang

E-mail: lgzhang@,zzu.edu.cn

Postal address: Henan Institute of Medical and Pharmaceutical Science, Zhengzhou University, No. 40 Daxue Road, Zhengzhou 450052, China

Tel: 086-17839908382

* Corresponding author: Liping Dai

E-mail: $1 p d @$ zzu.edu.cn

Postal address: Henan Institute of Medical and Pharmaceutical Science, Zhengzhou University, No. 40 Daxue Road, Zhengzhou 450052, China

Tel: 086-15290808333 
Table S1. Genetic sequences

\begin{tabular}{ccc}
\hline Gene & Sense & Antisense \\
\hline Bax & CCCGAGAGGTCTTTTTCCG & TGAGCACCAGTTTGCTGGC \\
Caspase3 & TGGTTCATCCAGTCGCTTTG & TTCTGTTGCCACCTTTCGG \\
GAPDH & GAACGGGAAGCTCACTGG & GCCTGCTTCACCACCTTCT \\
p53 & GTTGGTCGGTGGGTTGGTAGT & GGGATGGGGTGAGATTTCCTT \\
Piezo1 & GCGACACATAGGGGTCACAA & CAGACAGAGGAGACCACCAAGAT \\
\hline
\end{tabular}


Table S2. Antibodies used in the experiment with dilution ratios

\begin{tabular}{cc}
\hline Protein & Dilution ratio \\
\hline Bax (CST, USA) & $1: 1000$ \\
Caspase3 (CST,USA) & $1: 1000$ \\
Cleaved-caspase3 (CST,USA) & $1: 1000$ \\
GAPDH (CST,USA) & $1: 1000$ \\
p53 (CST,USA) & $1: 1000$ \\
Piezo1 (Abcam,USA) & $1: 500$ \\
E-cadherin (CST,USA) & $1: 1000$ \\
N-cadherin (CST,USA) & $1: 1000$ \\
Vinculin (CST,USA) & $1: 1000$ \\
\hline
\end{tabular}

